Feng-Ling Jin $M D$, Frances Chung FRCPC

\section{Postoperative pain - a challenge for anaesthetists in ambulatory surgery}

W

ITH the development of minimally invasive surgical procedures, ambulatory surgery in North America has grown remarkably over the last two decades. ${ }^{1}$ This growth is also attributed to the effort of anaesthetists to prevent and treat postoperative adverse outcomes with new drugs and techniques, thus ensuring the safety and satisfaction of patients. With these changes, a wider variety of patients and procedures are shifting into the ambulatory area, and the incidence of postoperative adverse outcomes will increase. Pain is one of the main postoperative adverse outcomes that causes distress to patients, ${ }^{2}$ prolongs their stay in the ambulatory care unit, ${ }^{3}$ and increases the incidence of unanticipated admission after surgery. ${ }^{4}$ How far have we gone in controlling postoperative pain? Are patients satisfied with the pain treatment they receive? What improvements can we make in the future?

In this issue, Beauregard and colleagues ${ }^{5}$ report on a study they conducted in a large teaching hospital on 89 ambulatory surgical patients to assess the intensity, duration, and impact of pain during their hospital stay and on the first, second and seventh postoperative days. More than $40 \%$ of the patients reported moderate to severe pain during their hospitalisation. Our study on 10,008 consecutive patients undergoing ambulatory surgery showed that severe pain was suffered by $5.3 \%$ of patients in the Postanaesthesia Care Unit and $1.3 \%$ of patients in the Ambulatory Surgery Unit. ${ }^{6}$ These results indicated that there was still a high incidence of severe pain after routine postoperative pain treatment during the hospital stay. In another study, a 24-hr telephone interview with the parents of 84 paediatric patients who underwent tonsillectomy indicated that pain control was inadequate in $25 \%$ of the patients. ${ }^{2}$ Beauregard and colleagues ${ }^{5}$ extended their investigation to the seventh postoperative day and found that the pain decreased with time but was still considerable. Postoperative pain affected the patients' return to normal functioning. These results indicated that routine management of post-discharge pain could not eliminate the pain completely. Effective postoperative pain control for patients with severe pain is needed, and take-home analgesia protocols need to be developed.

Some factors can predict the occurrence of postoperative pain. More men than women experience intense postoperative pain. 5,6 The patients with a higher body mass index had a high incidence of severe pain postoperatively, because the dose of opioid (fentanyl or alfentanil) administered during the surgical procedure was inadequate. ${ }^{6}$ The anaesthetist should pay more attention to increasing the dose of opioid properly during surgical procedures. In addition, lengthy surgical procedures and certain types of operations (orthopaedic, urological, general, plastic, neurosurgical, and ENT/dental) are predictors of severe pain. ${ }^{6}$

Successful postoperative pain control also depends on the knowledge and demands of the patient. A questionnaire for evaluating the general public's perception of postoperative pain revealed that almost $50 \%$ of patients were prepared to suffer pain rather than to complain. ${ }^{7}$ They knew little about postoperative pain and were confident in the ability of doctors and nurses to treat it. High satisfaction with a relatively high pain level was also found in Beauregard's study. ${ }^{5}$ Postoperative pain can have a deteriorative effect on the recovery of the patient from surgery, whereas multimodal balanced analgesia can accelerate postoperative rehabilitation and reduce hospital stay. Therefore, patient education is required for optimal pain management after ambulatory surgery. Patients should be given a full explanation of the need to minimise or reduce postoperative pain, and they should be informed of the various methods available to treat it. They should be assured that any pain that does occur will be properly and promptly treated.?

The continued training of doctors, nurses, and medical students in the prevention and proper management of postoperative pain is necessary. 8,9 Beauregard and colleagues $^{5}$ found that many instructions given to 
patients did not clearly indicate the intake frequency, dose adjustment, and control of side effects of analgesics. In the study of Rose et al., ${ }^{10}$ pain management practices were successfully changed after anaesthetists were educated and patients were made more aware of pain as an important problem. Another study showed that an audit of opioid prescribing considerably improved the prescribing of opioids, especially the frequency with which they were prescribed. ${ }^{11}$

An ideal postoperative analgesic protocol would be practical, effective, safe, have minimal side effects, and be cost-effective. ${ }^{12}$ Sustained-release morphine, ${ }^{13}$ synthetic opioid agonist-antagonist analgesics (butorphanol), ${ }^{14}$ nonsteroidal anti-inflammatory drugs (NSAID), patientcontrolled analgesia, ${ }^{15,16}$ local anaesthetic techniques (neuroaxial, ${ }^{17}$ intraarticular, ${ }^{18}$ nerve block ${ }^{19-21}$ ), and nonpharmacological techniques (transcutaneous acupoint electrical stimulation ${ }^{22}$ ) have broadened the choices for the management of postoperative pain. For reduction of opioid-related postoperative nausea and vomiting, the use of opioids and nonopioids combined with local anaesthetic techniques ${ }^{23,24}$ has been increasing. A randomised double-blind study of suprascapular nerve block for pain relief in arthroscopic shoulder surgery ${ }^{20}$ showed a $40 \%$ reduction in analgesic consumption and a reduction in pain at rest and on abduction. Another randomised double-blind study on preoperative multimodal analgesia for ambulatory patients who underwent laparoscopic cholecystectomy ${ }^{24}$ used a combination of preoperative meperidine and ketorolac by intramuscular bolus injection and local anaesthetic infiltration into the incision during laparoscopic cholecystectomy. The results showed that the concomitant use of local anaesthetic, NSAID, and opioid drugs was very effective in diminishing postoperative pain and reducing the stay in the ambulatory care unit. These new modalities demonstrate good effects on pain control and reduce the adverse outcomes related to surgery. The results encourage us to continue studies in this field.

Some new analgesic techniques are still controversial, and their clinical efficacy remains to be proved. ${ }^{21,25}$ The safety of some anaesthetic techniques should be further verified. ${ }^{21,26,27}$ For example, Bean-Lijewski ${ }^{21}$ reported two life-threatening cases of upper airway obstruction that resulted from glossopharyngeal nerve block for pain relief after paediatric tonsillectomy. New techniques must be evaluated with respect to cost, quality of recovery, duration of recovery, and postoperative adverse outcome.

In conclusion, severe postoperative pain in ambulatory surgical patients in the hospital and at home should not be underestimated. It is a challenge for anaesthetists to develop new analgesic techniques that are both safe and cost-effective, facilitate early ambulation, and do not increase the incidence of postoperative adverse outcomes.

\section{La douleur postopératoire - un défi pour les anesthésistes en chirurgie ambulatoire}

Avec le développement des procédures chirurgicales à effraction minime, nous avons assisté en Amérique du Nord, depuis deux décades, à un essor remarquable de la chirurgie ambulatoirel. Cette croissance est aussi attribuable aux efforts des anesthésistes pour prévenir et traiter les complications postopératoires au moyen de nouvelles techniques et médications, assurant ainsi la sécurité et la satisfaction des patients. À cause de ces changements, une plus grande variété de patients et de procédures prennent le chemin de l'ambulatoire et l'incidence des complications postopératoires va s'accroître. La douleur est une des complications postopératoires principales qui affecte les patients ${ }^{2}$, prolonge leur séjour à l'unité ambulatoire ${ }^{3}$, et augmente l'incidence des admissions non prévues après la chirurgie 4 . Où sommes-nous rendus dans le contrôle de la douleur postopératoire? Les patients sont-ils satisfaits des traitements reçus? Quelles améliorations futures devons-nous viser?

Dans ce numéro, Beauregard et collègues ${ }^{5}$ présentent une étude conduite dans un grand hôpital d'enseignement, portant sur 89 patients en chirurgie ambulatoire, et visant à évaluer l'intensité, la durée et les conséquences de la douleur durant leur séjour à l'hôpital et au cours des premier, second et septième jours postopératoires. Plus de $40 \%$ des patients font état de doulcur modérée à sévère durant leur hospitalisation. Notre étude portant sur 10,008 patients consécutifs en chirurgie ambulatoire a montré qu'une douleur sévère était le lot de $5,3 \%$ des patients à la salle de réveil et de $1,3 \%$ à l'unité de court séjour ${ }^{6}$. Ces résultats indiquent la persistance d'une incidence élevée de douleur sévère durant le séjour hospitalier, et ce malgré le traitement analgésique de routine. Dans une autre étude, une relance téléphonique à 24 heures des parents de 84 patients pédiatriques ayant subi une amygdalectomie a montré un soulagement inadéquat de la douleur dans 
$25 \%$ des cas $^{2}$. Beauregard et collègues 5 ont poursuivi leur étude jusqu'au $7^{\mathrm{c}}$ jour et ont trouvé que la douleur, même si elle s'atténue avec le temps, demeure significative. La douleur postopératoire prévient le retour à la normale des patients. Ces résultats démontrent que le traitement usuel de la douleur survenant après le congé n'élimine pas complètement cette douleur. Nous avons donc besoin de modalités de contrôle efficaces de la douleur postopératoire pour les patients avec douleur sévère et nous devons développer des protocoles pour l'analgésie à domicile.

Certains facteurs peuvent prédire la survenue de douleur postopératoire. Plus d'hommes que de femmes souffrent de douleur postopératoire intense $e^{5,6}$. Les patients avec un index de masse corporelle élevé ont présenté une incidence élevée de douleur postopératoire parce que la dose d'opiacé (fentanyl ou alfentanil) administré durant la chirurgie était inadéquate ${ }^{6}$. L'anesthésiste doit être attentif à augmenter de façon appropriée la dose d'opiacé durant la chirurgie. En outre, des procédures longues et certains types d'opérations (orthopédiques, urologiques, chirurgie générale, plastie, neurochirurgie et ORL/dentaire) font appréhender des douleurs sévères ${ }^{6}$.

Le contrôle efficace de la douleur postopératoire dépend aussi des connaissances et des attentes du patient. Un questionnaire destiné à évaluer la perception du grand public de la douleur postopératoire a révélé que près de $\mathbf{5 0} \%$ des patients étaient prêts à supporter la douleur plutôt que de se plaindre ${ }^{7}$. Ils connaissaient peu de choses concernant la douleur postopératoire et faisaient confiance aux médecins et aux infirmières pour la traiter. Un indice élevé de satisfaction malgré un indice élevé de douleur se retrouve aussi dans l'étude de Beauregard ${ }^{5}$. La douleur postopératoire peut avoir un effet négatif sur la récupération du patient après la chirurgie, alors qu'une analgésie balancée jouant sur plusieurs tableaux peut accélérer la récupération postopératoire et réduire le séjour hospitalier. Donc, l'éducation des patients est requise pour obtenir un traitement optimal de la douleur en chirurgie ambulatoire. On doit donner aux patients des explications complètes concernant la nécessité de réduire ou de minimiser la douleur postopératoire, et on doit les informer des diverses modalités de traitement. On doit les assurer que toute douleur sera traitée rapidement et de façon adéquate ${ }^{7}$.

Il faut continuer à former les médecins, les infirmières et les étudiants en médecine concernant la prévention et le traitement approprié de la douleur postopératoire ${ }^{8,9}$. Beauregard et collègues ${ }^{5}$ ont trouvé que plusieurs informations données aux patients n'indiquaient pas clairement la fréquence des prises, l'ajustement de la dose, et le mode de contrôle des effets secondaires des analgésiques. Dans l'étude de Rose $e t a l{ }^{10}$, les modalités de traitement de la douleur ont changé de façon efficace après formation des anesthésistes et après sensibilisation des patients à la notion de douleur comme problème sérieux. Une autre étude a montré que le fait de se pencher sur la prescription des opiacés améliore considérablement cette prescription, spécialement en ce qui concerne la fréquence d'administration ${ }^{11}$.

Un protocole idéal d'analgésie postopératoire serait pratique, efficace, sûr, aurait des effets secondaires minimes et serait rentable ${ }^{12}$. Les options de traitement de la douleur postopératoire se sont élargies et comprennent: la morphine à libération continue ${ }^{13}$, les analgésiques opiacés de type agoniste-antagoniste (butorphanol) ${ }^{14}$, les anti-inflammatoires non stérö̈diens (AINS), l'analgésic contrôlée par le patient ${ }^{15,16}$, les techniques d'anesthésie locale (neuraxial ${ }^{17}$, intra-articulaire ${ }^{18}$, différents blocs nerveux $\left.{ }^{19,21}\right)$, de même que les techniques non pharmacologiques (stimulation électrique transcutané de type acupoint ${ }^{22}$ ). En ce qui concerne la réduction des nausées et vomissements postopératoires reliés aux opiacés, l'usage des opiacés et des non opiacés combinés aux techniques d'anesthésie locale ${ }^{23,24} \mathrm{~s}$ 'est accru. Une étude aléatoire et à double insu d'un bloc suprascapulaire pour le soulagement de la douleur dans la chirurgie arthroscopique de l'épaule ${ }^{20}$ a montré une réduction de $40 \%$ de la consommation des analgésiques et une diminution de la douleur tant au repos qu'en abduction. Une autre étude aléatoire et à double insu sur l'analgésie multimode préopératoire chez des patients ambulatoires subissant une cholécystectomie laparoscopique ${ }^{24}$ a utilisé une combinaison préopératoire de mépéridine et de kétorolac par voie $I M$ associée à une infiltration d'anesthésique local dans la plaie durant la procédure. Les résultats ont montré que l'utilisation concomitante d'anesthésique local, d'AINS et d'opiacés était très efficace pour diminuer la douleur postopératoire et réduire la durée de séjour à l'unité de court séjour. Ces nouvelles modalités produisent de bons effets en termes de contrôle de la douleur et diminuent les complications liées à la chirurgie. Ces résultats nous encouragent dans nos recherches dans ce domaine.

Quelques nouvelles techniques analgésiques demeurent controversées, et leur efficacité clinique n'est pas encore prouvée ${ }^{21,25}$. La sécurité de certaines techniques anesthésiques doit être prouvée ${ }^{21,26,27}$. À titre d'exemple, Bean-Lijewski ${ }^{21}$ ont rapporté deux cas d'obstruction grave des voies aériennes supérieures résultant de bloc du nerf glosso-pharyngien pour le soulagement de la douleur après amygdalectomie chez des patients pédiatriques. Les nouvelles techniques doivent être évaluées quant au coût, à la qualité du réveil, à la durée du réveil ainsi qu'aux complications postopératoires. 
En conclusion, chez les patients en chirurgie ambulatoire, on ne doit pas sous estimer la douleur postopératoire sévère tant à l'hôpital qu'à domicile. C'est un défi pour les anesthésistes de mettre au point de nouvelles techniques analgésiques qui sont à la fois sûres et rentables, qui permettent un lever précoce et qui n'augmentent pas l'incidence de complications postopératoires.

\section{References}

1 White PF. Ambulatory anesthesia and surgery. past, present, and future. In: White PF (Ed.). Ambulatory Anesthesia \& Surgery, lst ed. London: W.B. Saunders Company Ltd, 1997: 3-34.

2 Sutters $K A$, Miaskowski $C$. Inadequate pain management and associated morbidity in children at home after tonsillectomy. J Ped Nurs 1997; 12: 178-85.

3 Chung F. Recovery pattern and home-readiness after ambulatory surgery. Anesth Analg 1995; 80: 896-902.

4 Fortier J, Chung F, Su J. Predictive factors of unanticipated admission in ambulatory surgery: a prospective study. Anesthesiology 1996; 85: A27.

5 Beauregard L, Pomp A, Choiniere M. Severity and impact of pain after day-surgery. Can J Anaesth 1998; 45: 304-11.

6 Chung F, Ritchie E, Su J. Postoperative pain in ambulatory surgery. Anesth Analg 1997; 85: 808-16.

7 Scott NB, Hodson M. Public perceptions of postoperative pain and its relief. Anaesthesia 1997; 52: 438-42.

8 Moote $C A$. The prevention of postoperative pain. Can J Anaesth 1994; 41: 527-33.

9 Smith $A F$. The prevention of postoperative pain: shouldn't it begin at medical school? (Letter) Can J Anaesth 1995; 42: 256-7.

10 Rose $D K$, Coben $M M, \Upsilon_{e e} D A$. Changing the practice of pain management. Anesth Analg 1997; 84: 764-72.

11 Humphries CA, Counsell DJ, Pediani RC, Close SL. Audit of opioid prescribing: the effect of hospital guidelines. Anaesthesia 1997; 52: 745-9.

$12 \mathrm{Joshi}$ GP. Postoperative pain management. In: White PF (Ed.). Ambulatory Anesthesia \& Surgery, lst ed. London: W.B. Saunders Company Ltd, 1997: 477-86.

13 Gourlay GK, Cherry $D A$, Onley $M M$, et al. Pharmacokinetics and pharmacodynamics of twentyfour-hourly Kapanol compared to twelve-hourly MS Contin in the treatment of severe cancer pain. Pain 1997; 69: 295-302.

14 Cannon $C R$. Transnasal butorphanol: pain relief in the head and neck patient. Otolaryngol Head Neck Surg 1997; 116: 197-200.

15 Wasylak T), Abbott FV, English MJM, Jeans ME. Reduction of postoperative morbidity following patient-controlled morphine. Can J Anaesth 1990; 37: 726-31.
16 Striebel HW, Oelmann $T$, Spies $C$, Rieger A, Schwagmeier $R$. Patient-controlled intranasal analgesia: a method for noninvasive postoperative pain management. Anesth Analg 1996; 83: 548-51.

17 Fuller JG, McMorland GH, Douglas MJ, Palmer L. Epidural morphine for analgesia after Caesarean section: a report of 4880 patients. Can J Anaesth 1990; 37: 636-40.

18 Reuben SS, Connelly NR. Postoperative analgesia for outpatient arthroscopic knee surgery with intraarticular bupivacaine and ketorolac. Anesth Analg 1995; 80: 1154-7.

19 Bourke DL, Furman WR. Improved postoperative analgesia with morphine added to axillary block solution. J Clin Anesth 1993; 5: 114-7.

20 Ritchie ED, Tong D, Chung F, Norris AM, Miniaci A, Vairavanathan $S D$. Suprascapular nerve block for postoperative pain relief in arthroscopic shoulder surgery: a new modality? Anesth Analg 1997; 84: 1306-12.

21 Bean-Lijewski JD. Glossopharyngeal nerve block for pain relief after pediatric tonsillectomy: retrospective analysis and two cases of life-threatening upper airway obstruction from an interrupted trial. Anesth Analg 1997; 84: 1232-8.

22 Wang B, Tang J, White PF, et al. Effect of the intensity of transcutaneous acupoint electrical stimulation on the postoperative analgesic requirement. Anesth Analg 1997; 85: 406-13.

23 Marquardt HM, Razis $P A$. Prepacked take-home analgesia for day case surgery. Br J Nurs 1996; 5: 1114-8.

24 Michaloliakou C, Chung F, Sharma S. Preoperative multimodal analgesia facilitates recovery after ambulatory laparoscopic cholecystectomy. Anesth Analg 1996; 82: 44-51.

25 Badner NH, Bourne RB, Rorabeck CH, Doyle JA. Addition of morphine to intra-articular bupivacaine does not improve analgesia following knee joint replacement. Reg Anesth 1997; 22: 347-50.

26 Pande AC, Pyke RE, Greiner M, Wideman GL, Benjamin R, Pierce $M W$. Analgesic efficacy of enadoline versus placebo or morphine in postsurgical pain. Clin Neuropharmacol 1996; 19: 451-6.

27 Looi-Lyons LC, Chung FF, Chan VW, McQuestion M. Respiratory depression: an adverse outcome during patient controlled analgesia therapy. J Clin Anesth 1996; 8: 151-6. 